\title{
Clinicopathological Findings and Imaging Features of Intraductal Papillary Neoplasm of the Bile Ducts
}

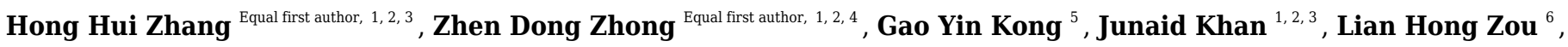 \\ Yu Jiang ${ }^{6}$, Xie Hong Liu ${ }^{6}$, Yi Xun Tang ${ }^{5}$, Bo Jiang ${ }^{1,2,3}$, Chuang Peng ${ }^{1,2,3}$, Ying Hui Song ${ }^{\text {Corresp., 1, 2,3 }}$, su lai liu ${ }^{\text {Corresp. 1, 2, } 3}$ \\ ${ }^{1}$ Department of Hepatobiliary Surgery/Hunan Research Center of Biliary Disease, Hunan Provincial People's Hospital/The First Affiliated Hospital of Hunan \\ Normal University, Changsha, Hunan province, China \\ 2 Biliary Disease Research Laboratory of Hunan Provincial People's Hospital, Key Laboratory of Hunan Normal University, Changsha, Hunan province, \\ China \\ 3 \\ ${ }^{3}$ Clinical Medical Technology Research Center of Hunan Provincial for Biliary Disease Prevention and Treatment, Changsha, Hunan province, China \\ Department of Hepatobiliary Surgery, Changsha County People's Hospital/Hunan Provincial People's Hospital Xingsha Campus, Changsha, Hunan \\ province, China \\ 5 Department of Anesthesiology, Hunan Provincial People's Hospital/Clinical Research Center for Anesthesiology of ERAS in Hunan Province, Changsha, \\ Hunan province, China \\ 6 Key Laboratory of Study and Discovery of Small Targeted Molecules of Hunan Province, School of Medicine, Hunan Normal University/Hunan Provincial \\ Institute of Emergency, Changsha, Hunan province, China \\ Corresponding Authors: Ying Hui Song, su lai liu \\ Email address: sissysyh@163.com, liusulai@hunnu.edu.cn
}

Background. Intraductal papillary mucinous neoplasm of the bile duct (IPMN-B) is considered an uncommon tumor, and there is limited understanding of IPMN-B. This study aimed to investigate the prognosis and influential factors of the IPMN-B from 58 cases.

Methods. The clinical data of 58 patients with pathologically confirmed IPMN-B admitted to our hospital from January 1, 2012 to August 2017 were collected and analyzed. The patients were followed up by outpatient or telephone until January 1, 2019. SPSS19.0 software was applied for data analysis. Survival analysis was performed using Kaplan-Meier method and parallel Log-rank test. Prognostic factors were analyzed by univariate analysis and multiple Cox regression model.

Results. Among of all the patients, 26 cases were benign tumors and 32 cases were malignant tumors. The preoperative tumor markers CA242 and CEA of malignant IPNM-B patients were significantly higher than those in benign tumors $(P<0.05)$. Survival analysis showed that patients with malignant tumors had a worse prognosis. The median survival time of malignant IPMN-B patients was $40.6 \pm 3.0$ months, yet median survival time of benign IPMN-B patients was not reached $(P=0.19)$. The one-year survival rate and three-year survival rate of benign IPMN-B were $84 \%$ and $74 \%$ respectively. The one-year survival rate and three-year survival rate of malignant IPMN-B were $88 \%$ and $64 \%$ respectively.

Univariate analysis showed that combined lymph node metastasis, surgical method, and differentiation degree could affect patients' prognosis $(P<0.05)$. Multivariate analysis showed differentiation degree was an independent risk factor affecting prognosis $(O R=0.06,95 \%$ confidence interval: $0.007 \sim 0.486, P$ $<0.05)$.

Conclusion. The levels of CEA and CA242 were helpful to identify benign and malignant of IPNM-B. Moreover, radical surgical resection could prolong patients' survival. Finally, differentiation degree was an independent risk factor affecting malignant IPNM-B prognosis. 


\section{Clinicopathological Findings and Imaging Features of}

\section{Intraductal Papillary Neoplasm of the Bile Ducts}

3 Honghui Zhang ${ }^{1,2,3 \#}$, Zhendong Zhong ${ }^{1,2,4 \#}$, Gaoyin Kong ${ }^{5}$, Junaid Khan ${ }^{1,2,3}$, Lianhong Zou ${ }^{6}$, Yu

4 Jiang $^{6}$, Xiehong Liu ${ }^{6}$, Yixun Tang ${ }^{5}$, Bo Jiang ${ }^{1,2,3}$, Chuang Peng ${ }^{1,2,3}$, Yinghui Song ${ }^{1,2,3 *}$,Sulai

$5 \mathrm{Liu}^{1,2,3 *}$

$6{ }^{1}$ Department of Hepatobiliary Surgery/Hunan Research Center of Biliary Disease, Hunan

7 Provincial People's Hospital/The First Affiliated Hospital of Hunan Normal University,

8 Changsha, 410005 Hunan Province, China

9 ²Biliary Disease Research Laboratory of Hunan Provincial People's Hospital, Key Laboratory of

10 Hunan Normal University, Changsha, 410005 Hunan Province, China

$11{ }^{3}$ Clinical Medical Technology Research Center of Hunan Provincial for Biliary Disease

12 Prevention and Treatment, Changsha, 410005 Hunan Province, China

$13{ }^{4}$ Department of Hepatobiliary Surgery, Changsha County People's Hospital/Hunan Provincial

14 People's Hospital Xingsha Campus, Changsha, China.

$15{ }^{5}$ Department of Anesthesiology, Hunan Provincial People's Hospital/Clinical Research Center

16 for Anesthesiology of ERAS in Hunan Province, Changsha 410005, China

$17{ }^{6}$ Key Laboratory of Study and Discovery of Small Targeted Molecules of Hunan Province,

18 School of Medicine, Hunan Normal University/Hunan Provincial Institute of Emergency,

19 Changsha, Hunan province, China

20 \#This author contributes equally to this work.

$21 *$ Corresponding author:

22 1)Yinghui Song PhD, M. D.; 
23 2) Sulai Liu,PhD, M.D.

24 *This author contributes equally to this work.

25 Address: Department of Hepatobiliary Surgery, Hunan Provincial People's Hospital/The First

26 Affiliated Hospital of Hunan Normal University, Changsha, Hunan Province, People's Republic

27 of China.

28 Tel/fax: 08673183929520.

29 E-mail: **sissysyh@163.com and liusulai@hunnu.edu.cn

\section{Abstract}

31 Background. Intraductal papillary mucinous neoplasm of the bile duct (IPMN-B) is considered

32 an uncommon tumor, and there is limited understanding of IPMN-B. This study aimed to

33 investigate the prognosis and influential factors of the IPMN-B from 58 cases.

34 Methods. The clinical data of 58 patients with pathologically confirmed IPMN-B admitted to our

35 hospital from January 1, 2012 to August 2017 were collected and analyzed. The patients were

36 followed up by outpatient or telephone until January 1, 2019. SPSS19.0 software was applied for

37 data analysis. Survival analysis was performed using Kaplan-Meier method and parallel Log-rank

38 test. Prognostic factors were analyzed by univariate analysis and multiple Cox regression model.

39 Results. Among of all the patients, 26 cases were benign tumors and 32 cases were malignant

40 tumors. The preoperative tumor markers CA242 and CEA of malignant IPNM-B patients were

41 significantly higher than those in benign tumors $(\mathrm{P}<0.05)$. Survival analysis showed that patients

42 with malignant tumors had a worse prognosis. The median survival time of malignant IPMN-B

43 patients was $40.6 \pm 3.0$ months, yet median survival time of benign IPMN-B patients was not

44 reached $(\mathrm{P}=0.19)$. The one-year survival rate and three-year survival rate of benign IPMN-B were

$4584 \%$ and $74 \%$ respectively. The one-year survival rate and three-year survival rate of malignant 
46 IPMN-B were $\quad 88 \% \quad$ and $\quad 64 \%$ respectively.

47 Univariate analysis showed that combined lymph node metastasis, surgical method, and 48 differentiation degree could affect patients' prognosis $(\mathrm{P}<0.05)$. Multivariate analysis showed 49 differentiation degree was an independent risk factor affecting prognosis $(\mathrm{OR}=0.06,95 \%$ 50 confidence interval: $0.007 \sim 0.486, \mathrm{P}<0.05)$.

51 Conclusion. The levels of CEA and CA242 were helpful to identify benign and malignant of 52 IPNM-B. Moreover, radical surgical resection could prolong patients' survival. Finally, 53 differentiation degree was an independent risk factor affecting malignant IPNM-B prognosis.

54 Keywords: Papillary mucinous tumor in the bile duct; radical resection; prognosis; risk factor 55 Introduction Intraductal papillary mucinous neoplasm of the bile duct (IPMN-B) is considered an uncommon tumor, which secretes a large amount of mucus to cause significant expansion and obstruction of the bile ducts. Kim HJ, et al. described nine cases of bile duct tumors secreting a large amount of mucus and concluded that their clinical, imaging and pathological features were similar to those of papillary mucinous tumors in the pancreatic duct in 2000(Kim HJ et al., 2000). In 2008, the concept of IPMN-B was first proposed to summarize such diseases (Paik KY et al., 2008). There is limited understanding of IPMN-B, which are mostly case reports, and lack of large-scale clinical research (Nakanuma Y et al.,2016; Hokuto D et al., 2017). This study retrospectively analyzed 58 patients with IPMN-B admitted in our hospital from January 2012 to August 2017. Clinical and pathological data were collected and explored to identify the prognosis and influencing factors of IPMN-B in order to provide basis for the clinical diagnosis and treatment of IPMN-B.

\section{Clinical manifestations}


69 The clinical data of 58 patients with postoperative pathological diagnosis of IPMN-B were

70 collected, including 24 males and 34 females; the median age was 61 years (41-85 years). The

71 clinical manifestations were mainly pain in the right upper abdomen with jaundice. Abdominal

72 ultrasound, CT, or MRI examinations were performed before surgery. Typical imaging findings

73 were local or intrahepatic and extrahepatic bile duct dilatation, thickening of the bile duct wall

74 with nodular tissue protrusions. Jelly-like bile was seen on duodenoscope examination in some

75 patients. The study was approved by the Ethics Committee of Hunan Provincial People's

76 Hospital/The First Affiliated Hospital of Hunan Normal University (Number: 2020-11), and all

77 clinical samples were used in accordance with institutional guidelines and the Declaration of

78 Helsinki after obtaining signed informed consent from all participants.

79 Surgical methods

80 Preoperative auxiliary examinations were performed to evaluate important organ functions, liver

81 reserve functions, and resectability of liver. Pathological histological examinations were

82 performed during and after surgery. According to the results of pathological histological

83 examinations and the degree of tumor invasion, they were divided into three subgroups: atypical

84 hyperplasia, adenoma and adenocarcinoma (including cancerous changes). The first two groups

85 belonged to benign tumors. The surgical method was hepatic resection or combined tail resection

86 according to the tumor location, and the intraoperative pathological results. The malignant tumors

87 were routinely dissected with lymph nodes in groups $8,12,13$ a. Patients with multiple intrahepatic

88 bile duct tumors by intraoperative choledochoscopy underwent hepatobiliary reconstruction, bile-

89 gut drainage, and T-tube drainage. The specimens submitted including resected liver specimen, 90 resection margins and lymph nodes.

$91 \quad$ Follow up 
92 The patients' gender, age, jaundice, biliary stones, previous biliary surgery, tumor markers (CA19-

93 9, CA242, CEA), histological characteristics, lymph node metastasis, resection margins, etc. were

94 followed up in outpatient and telephone visits after the operation. Recurrence was defined as both

95 the recurrence of jaundice and new lesions on imaging. The end point of follow-up was the death

96 of patients or the follow-up time until January 1, 2019, and those who died for other reasons but

97 not IPMN-B were excluded.

98 Statistical methods

99 SPSS19.0 software was applied for data analysis. Categorical data was compared using

100 frequencies expressed as percentages, and compared with chi-squared testing. Tumor markers

101 were analyzed by variance among subgroups. Overall survival analysis was performed using

102 Kaplan-Meier method and parallel Log-rank test. Prognostic factors were analyzed by univariate

103 analysis and multiple Cox regression model. $\mathrm{P}<0.05$ was considered statistically significant.

\section{Results}

\section{Clinicopathological parameters of IPNM-B patients}

10645 patients (77.6\%) had bile duct stones, 35 patients (60.3\%) had previous biliary surgery, and 38

107 patients (65.5\%) had jaundice. Six of these 38 patients underwent percutaneous liver puncture of

108 the biliary tract to remove jelly-like bile. The pathological results showed 14 cases $(24.1 \%)$ were

109 atypical hyperplasia, 12 cases (20.7\%) were adenoma, and 32 cases (55.2\%) were adenocarcinoma

110 (including canceration). Lymph node metastasis did not occur in benign tumors, and lymph node

111 metastasis occurred in 6 cases (18.8\%) of malignant tumors. Radical resection was performed in

11246 cases (79.3\%), and palliative resection was performed in 12 cases (20.7\%). Malignant tumor

113 differentiation grade: 17 cases with high differentiation (53.1\%), 9 cases with high-medium

114 differentiation or middle differentiation (28.1\%), 6 cases with medium-low differentiation or poor 
115 differentiation (18.8\%). It was reported that CA19-9, CA242 and CEA were three recognized

116 plasma tumor markers of cholangiocarcinoma (Wongkham S et al., 2012). In this study, all of the

117 three biomarkers in adenocarcinoma group were higher than the other two groups $(\mathrm{P}<0.05)$. See

118 Table 1 for details. Moreover, CA242 and CEA were more valuable in the diagnosis of

119 cholangiocarcinoma (Ni XG et al.,2005). In this study, the area under the CA242 curve was 0.781 ,

120 meanwhile, the area under the CEA curve was $0.728(\mathrm{P}>0.05)$. It seemed that CA242 was more

121 sensitive and specific than CEA in diagnosing malignant IPMN-B from the ROC curve (Figure 1).

122 All patients in this study underwent radical or palliative surgical treatment. Among them, 13

123 patients who underwent T-tube drainage and palliative intestinal drainage due to acute obstructive

124 suppurative cholangitis (AOSC) or multiple tumors had a shorter survival time than those who

125 underwent liver resection $(p<0.05)$. The median survival time of patients who achieved R0

126 resection was significantly longer than those of $\mathrm{R} 1, \mathrm{R} 2$ resection, meanwhile the recurrence rate

127 of patients with R1, R2 resection was significantly higher than that of R0 resection (R0 is complete

128 microscopic resection, R1 is residual microscopic disease and R2 is residual macroscopic disease).

129 Imaging of IPNM-B

130 In this study, massive jelly-like mucus could be seen in the bile duct drainage of most patients.

131 Typical CT manifestations showed obvious cystic dilatation of the intrahepatic bile duct, a small

132 spot-shaped high-density lesion in the bile duct of the right hepatic lobe (Figure 2A). T2 magnetic

133 resonance imaging showed a clear soft vine-like expansion of the intrahepatic bile duct, and a

134 small nodular T2 signal foci in the bile duct (Figure 2B). The duodenoscopy showed that a

135 duodenal fistula with a diameter of $1.0 \mathrm{~cm}$ above the nipple which is filled with jelly-like slime

136 (Figure 2C).

\section{Survival analysis}


13851 cases $(87.9 \%)$ were followed up to the endpoint, no perioperative deaths, the average follow-

139 up time of these patients was $30.0 \pm 3.2$ months. Four patients with benign tumors died, of which

140 two elderly patients survived 3.7 months and 8.7 months, respectively; two patients survived 7.5

141 months and 35.2 months due to multiple intrahepatic and extrahepatic bile ducts lesions,

142 respectively. The overall mean survival time of this group was $56.4 \pm 6.0$ months. 10 patients with

143 malignant tumors died, the median overall survival time was $41.4 \pm 6.1$ months. Kaplan-Meier

144 survival curves of two groups were shown in Figure 3. Log-rank test $=1.65, P=0.19$. The one-

145 year survival rate and three-year survival rate of benign IPMN-B were $84 \%$ and $74 \%$ respectively.

146 The one-year survival rate and three-year survival rate of malignant IPMN-B were $88 \%$ and $64 \%$

147 respectively. Univariate Cox proportional hazard regression analyses showed that lymph node

148 metastasis, surgical method, and degree of differentiation were prognostic factors of malignant

149 IPNM-B $(\mathrm{P}<0.05)$. See Table 2 for details. Including lymph node metastasis, surgical method, and

150 degree of differentiation into the COX model and performing multivariate analysis showed that

151 differentiation degree $(\mathrm{OR}=0.06,95 \%$ confidence interval: $0.007-0.486, \mathrm{P}<0.05)$ was an

152 independent factor affecting prognosis.

\section{Discussion}

154 IPMN-B is one of intraductal papillary neoplasm of the bile duct. It secretes a large amount of mucus to block the bile duct and causes obstructive jaundice. Histopathology of IPMN-B is similar to intraductal papillary mucinous neoplasm of the pancreas (IPMN-P) (Nakanuma Y et al., 2017;

157 Fukumura $Y$ et al., 2017). However, it is also found that IPNB arising in intrahepatic catheters is 158 the biliary counterpart of IPMN, while IPNB arising in extrahepatic catheters is different from 159 typical IPMN (Yasuni Nakanuma et al., 2019). Most patients experienced preoperative ultrasound 160 examination showed significant bile duct dilatation, hypoechoic flocculent light masses in the bile 
161 duct, and obvious space occupying. Typical CT or MRI imaging features were soft vine-like

162 dilatation of the bile ducts inside and outside the liver, and multiple nodules in the bile duct wall.

163 Previous studies have suggested that CT play an important role in identifying benign and malignant

164 diseases of IPMN-B (Oki H et al., 2011; Paik KY et al., 2008). It is reported that "floating sign" in

165 the bile duct of MRI imaging is a typical manifestation of IPMN-B (Ying SH et al., 2015). In

166 general, the imaging characteristics of IPMN-B patients in this study were basically consistent

167 with the reported imaging characteristics.

168 The tumor markers CA242 and CEA of most patients were significantly increased before surgery,

169 and they were helpful to distinguish benign from malignant diseases. It seemed that CA242 was

170 more sensitive and specific than CEA in diagnosing malignant IPMN-B in this study, however,

171 this conclusion needed more samples to verify. It is more credible that combination all the three

172 biomarkers to assess the benign and malignant disease.

173 IPMN-B often presents with multiple lesions. Intrahepatic bile duct is the most common site and

174 accounts for about $84 \%$ of all the IPMN-B. When intrahepatic and extrahepatic bile duct tumors

175 invaded to the hilar region often indicated an enhancement of tumor infiltration. Although the

176 progress of bile duct and pancreatic papillary adenomas is relatively slow, the prognosis is often

177 poor when multiple infiltrates appear (Luvira V et al., 2016).

178 Radical surgical resection is considered the only way to cure bile duct malignancies. Even the

179 addition of EGFR-mAbs to gemcitabine-based first-line chemotherapy dose not significantly

180 improve neither overall survival nor progression-free survival (Rizzo A et al., 2020). All patients

181 in this study underwent radical or palliative surgical treatment.

182 The original cells of IPNB are considered to be bile duct gland cells, which are distributed along

183 the intrahepatic bile duct and extrahepatic bile ducts, showing a slow transition from adenoma to 
184 adenocarcinoma with fewer lymph nodes or distant metastases. The prognosis of IPMN-B is

185 significantly better than any other types of cholangiocarcinoma (Schlitter AM et al., 2014; Gordon

186 Weeks AN et al., 2016). In this study, IPMN-B often accompanied by biliary stones and it

187 progressed slowly, but once jaundice appeared, it was easy to induce AOSC. So it is necessary for

188 patients with stones or previous history of biliary surgery to perform routine physical examination.

189 Radical surgery could effectively improve the survival rate of patients with IPMN-B. The role of 190 adjuvant treatment for IPMN-B is yet to be established (Yeh TS et al., 2006). Therefore, it is

191 particularly essential to take the optimal surgical method. For multiple tumors involving the hilar

192 area, in order to avoid the occurrence of biliary obstruction, bile-intestinal drainage may be

193 necessary to increase the diameter of bile outflow channels.

194 In this study, there was different of the cumulative survival rate between benign IPMN-B patients 195 and malignant IPMN-B patients. The median survival time of malignant IPMN-B patients was $19640.6 \pm 3.0$ months, yet median survival time of benign IPMN-B patients was not reached $(\mathrm{P}=0.19)$,

197 the survival of patients in the malignant group was worse than that of the benign group, which was 198 consistent with the previous studies of IPMN-B survival time (Wang X et al., 2015; Luvira V et $199 a l ., 2017)$. But the difference between the two groups is not significant. It is considered that the 200 number of cases in this study is small and the survival time of two older patients in the benign 201 group is particularly short. In the future, we will continue to follow up patients with this disease in 202 order to obtain more convincing data to gain a deeper understanding of IPMN.

\section{Conclusions}

204 In short, preoperative CT and MRI is helpful to improve the detection rate of IPMN-B. Meanwhile, 205 the levels of CEA and CA242 are helpful to identify benign and malignant of IPNM-B. Moreover, 206 radical surgical resection could prolong patients' survival, when radical surgery is not available, 
207 unobstructed drainage is necessary. Finally, more data should be collected from more patients of

208 IPMN-B and long-term survival follow-up to improve the diagnosis and treatment of IPMN-B.

\section{Acknowledgements}

210 This work was financially supported by following funds: Central Guidance of Local Science and

211 Technology Development Fund (Grant No. 2018CT5008)/ Project of Scientific Research of

212 Traditional Chinese Medicine in Hunan (Grant No. 201809)/ Hunan Provincial Natural Science

213 Foundation of China (Grant No. 2019JJ50320 /2018JJ3296)/ Clinical Research Center for

214 Anesthesiology of ERAS in Hunan Province (No. 2018SK7001).

\section{Conflict of interest}

216 The authors declare that they have no financial or commercial conflict of interest.

\section{References}

218

219

220

221

222

223

224

225

226

227

228

Kim HJ, Kim MH, Lee SK, Yoo KS, Park ET, Lim BC, Park HJ, Myung SJ, Seo DW, Min YI. 2000. Mucin-hypersecreting bile duct tumor characterized by a striking homology with an intraductal papillary mucinous tumor (IPMT) of the pancreas. Endoscopy 32:389-93. DOI: $10.1055 / \mathrm{s}-2000-8996$.

Paik KY, Heo JS, Choi SH, Choi DW. 2008. Intraductal papillary neoplasm of the bile ducts: the clinical features and surgical outcome of 25 cases. J Surg Oncol 97:508-512. DOI: $10.1002 /$ jso.20994.

Nakanuma Y, Kakuda Y, Uesaka K, Miyata T, Yamamoto Y, Fukumura Y, Sato Y, Sasaki M, Harada K, Takase M.2016. Characterization of intraductal papillary neoplasm of bile duct with respect to histopathologic similarities to pancreatic intraductal papillary mucinous neoplasm. Hum Pathol 51: 103-113. DOI: 10.1016/j.humpath.2015.12.022. 
229

230

231

232

233

234

235

236

237

238

Hokuto D, Nomi T, Yasuda S, Yoshikawa T, Ishioka K, Yamada T, Akahori T, Nakagawa K, Nagai M, Nakamura K, Obara S, Kanehiro H, Sho M. 2017. Long-term observation and treatment of a widespread intraductal papillary neoplasm of the bile duct extending from the intrapancreatic bile duct to the bilateral intrahepatic bile duct: A case report. Int J Surg Case Rep 38: 166-171. DOI: 10.1016/j.ijscr.2017.07.031.

Wongkham S, Silsirivanit A. 2012. State of serum markers for detection of cholangiocarcinoma. Asian Pac J Cancer Prev 13:17-27.

Ni XG, Bai XF, Mao YL, Shao YF, Wu JX, Shan Y, Wang CF, Wang J, Tian YT, Liu Q, Xu DK, Zhao P. 2005. The clinical value of serum CEA, CA19-9, and CA242 in the diagnosis and prognosis of pancreatic cancer. Eur $J$ Surg Oncol 31:164-169. DOI: $10.1016 /$ j.ejso.2004.09.007.

Nakanuma Y, Uesaka K, Miyayama S, Yamaguchi H, Ohtsuka M. 2017. Intraductal neoplasms of the bile duct. A new challenge to biliary tract tumor pathology. Histol Histopathol 32: 1001-1015. DOI: 10.14670/HH-11-892.

Fukumura Y, Nakanuma Y, Kakuda Y, Takase M, Yao T. 2017. Clinicopathological features of intraductal papillary neoplasms of the bile duct: a comparison with intraductal papillary mucinous neoplasm of the pancreas with reference to subtypes. Virchows Arch 471: 65-76. DOI: $10.1007 / \mathrm{s} 00428-017-2144-9$.

Nakanuma Y, Kakuda Y, Uesaka K. 2019. Characterization of Intraductal Papillary Neoplasm of the Bile Duct with Respect to the Histopathologic Similarities to Pancreatic Intraductal Papillary Mucinous Neoplasm. Gut Liver 13:617-627. DOI: 10.5009/gnl18476

Oki H, Hayashida Y, Namimoto T, Aoki T, Korogi Y, Yamashita Y. 2011. Usefulness of gadolinium-ethoxybenzyl-diethylenetriamine pentaacetic acid-enhanced magnetic resonance 
252

253

254

255

256

257

258

259

260

261

262

263

264

265

266

cholangiography for detecting mucin retention in bile ducts: a rare intraductal papillary mucinous neoplasm of the bile duct. Jpn J Radiol 29:590-594. DOI: 10.1007/s11604-0110593-7.

Ying SH, Teng XD, Wang ZM, Wang QD, Zhao YL, Chen F, Xiao WB. 2015. Gd-EOBDTPA-enhanced magnetic resonance imaging for bile duct intraductal papillary mucinous neoplasms. World J Gastroenterol 21:7824-7833. DOI: 10.3748/wjg.v21.i25.7824.

Wang X, Cai YQ, Chen YH, Liu XB. 2015. Biliary tract intraductal papillary mucinous neoplasm: report of 19 cases. World $J$ Gastroenterol 21:4261-4267. DOI: $10.3748 /$ wjg.v21.i14.4261.

Luvira V, Pugkhem A, Tipwaratorn T, Chamgramol Y, Pairojkul C, Bhudhisawasdi V. 2016. Simultaneous Extensive Intraductal Papillary Neoplasm of the Bile Duct and Pancreas: A Very Rare Entity. Case Rep Surg 2016: 1518707. DOI: 10.1155/2016/1518707.

Rizzo A, Frega G, Ricci AD, Palloni A, Abbati F, DE Lorenzo S, Deserti M, Tavolari S, Brandi G. 2020. Anti-EGFR Monoclonal Antibodies in Advanced Biliary Tract Cancer: A Systematic Review and Meta-analysis. In Vivo 34:479-488. DOI:10.21873/invivo.11798.

Schlitter AM, Born D, Bettstetter M, Specht K, Kim-Fuchs C, Riener MO, Jeliazkova P, Sipos B, Siveke JT, Terris B, Zen Y, Schuster T, Höfler H, Perren A, Klöppel G, Esposito I. 2014. Intraductal papillary neoplasms of the bile duct: stepwise progression to carcinoma involves common molecular pathways. Mod Pathol 27: 73-86. DOI: $10.1038 /$ modpathol.2013.112.

Gordon-Weeks AN, Jones K, Harriss E, Smith A, Silva M. 2016. Systematic Review and Metaanalysis of Current Experience in Treating IPNB: Clinical and Pathological Correlates. Ann Surg 263: 656-63. DOI: 10.1097/SLA.0000000000001426. 
275 Yeh TS, Tseng JH, Chiu CT, Liu NJ, Chen TC, Jan YY, Chen MF. 2006. Cholangiographic

276 spectrum of intraductal papillary mucinous neoplasm of the bile ducts. Ann Surg 244:248-53.

277 DOI: $10.1097 / 01$. sla.0000217636.40050.54.

278 Luvira V, Pugkhem A, Bhudhisawasdi V, Pairojkul C, Sathitkarnmanee E, Luvira V,

279 Kamsa-Ard S. 2017. Long-term outcome of surgical resection for intraductal papillary

280 neoplasm of the bile duct. J Gastroenterol Hepatol 32: 527-533. DOI: 10.1111/jgh.13481.

281 


\section{Figure legend}

283 Figure 1 ROC curve of CA242 and CEA

284 Figure 2 Imaging of IPNM-B: (A)Typical CT manifestations showed obvious cystic dilatation of 285 the intrahepatic bile duct, a small spot-shaped high-density lesion in the bile duct of the right 286 hepatic lobe. (B) T2 magnetic resonance imaging showed a clear soft vine-like expansion of the 287 intrahepatic bile duct, and a small nodular T2 signal foci in the bile duct. (C)The duodenoscopy 288 showed a common bile duct duodenal fistula by below the duodenum above the duodenal papilla, 289 about $1.0 \mathrm{~cm}$ in diameter, full filled with full of jelly-like Slime.

290 Figure 3 The cumulative survival of patients with benign IPNM-B patients and malignant IPNM291 B patients was determined by the Kaplan-Meier method. 
Figure 1

Figure 1

ROC curve of CA242 and CEA

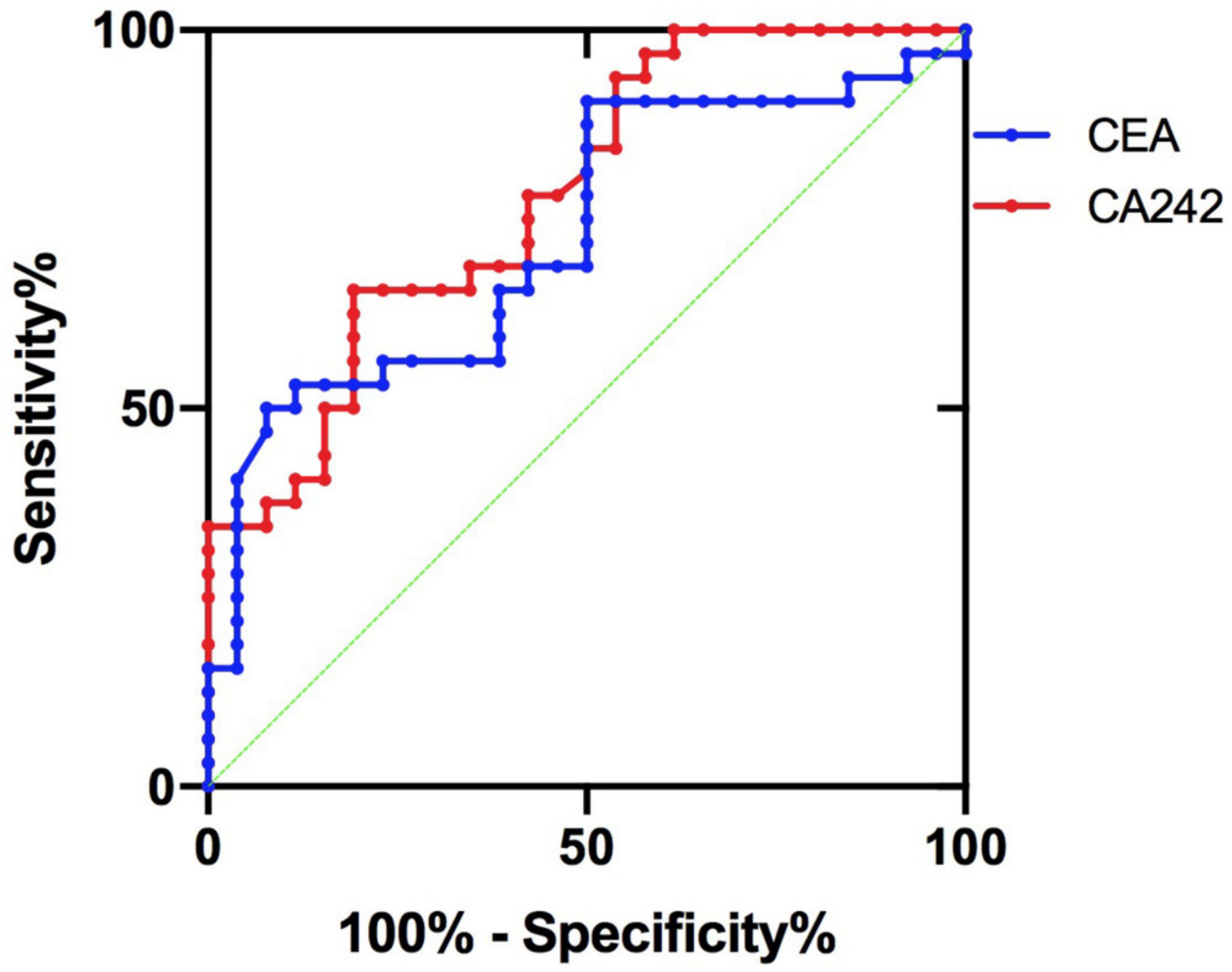




\section{Figure 2}

Figure 2

Imaging of IPNM-B: (A)Typical CT manifestations showed obvious cystic dilatation of the intrahepatic bile duct, a small spot-shaped high-density lesion in the bile duct of the right hepatic lobe. (B) T2 magnetic resonance imaging showed a clear soft vine-like expansion of the intrahepatic bile duct, and a small nodular T2 signal foci in the bile duct. (C)The duodenoscopy showed a common bile duct duodenal fistula by below the duodenum above the duodenal papilla, about $1.0 \mathrm{~cm}$ in diameter, full filled with full of jelly-like Slime.
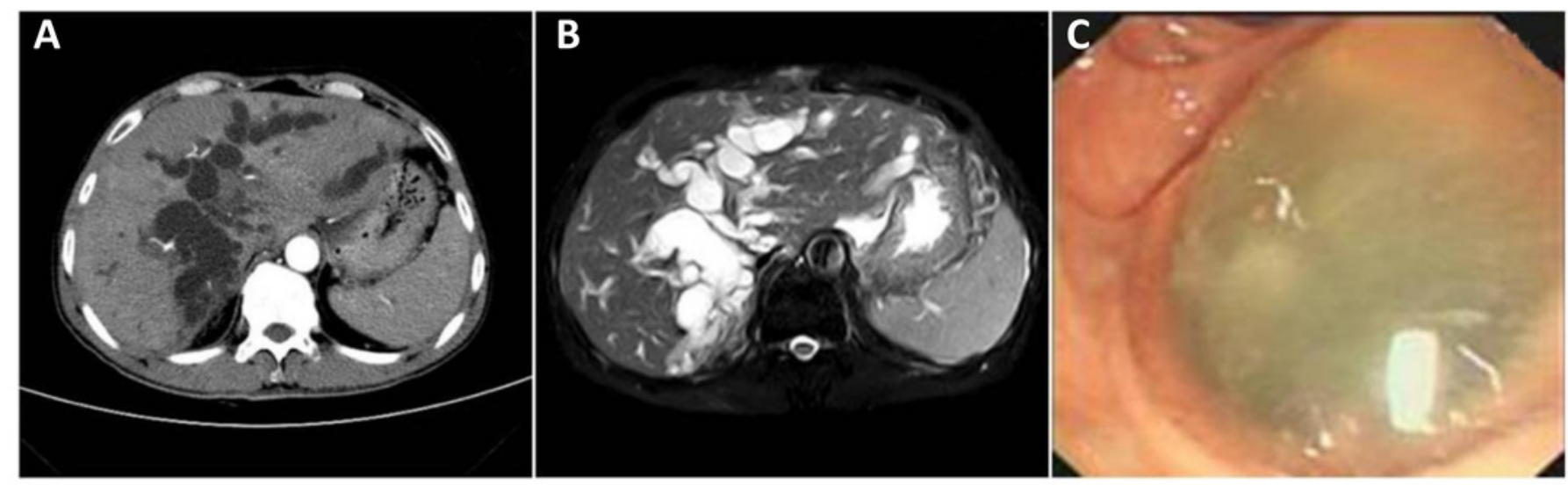
Figure 3

Figure 3

Figure 3 The cumulative survival of patients with benign IPNM-B patients and malignant IPNM-B patients was determined by the Kaplan-Meier method.

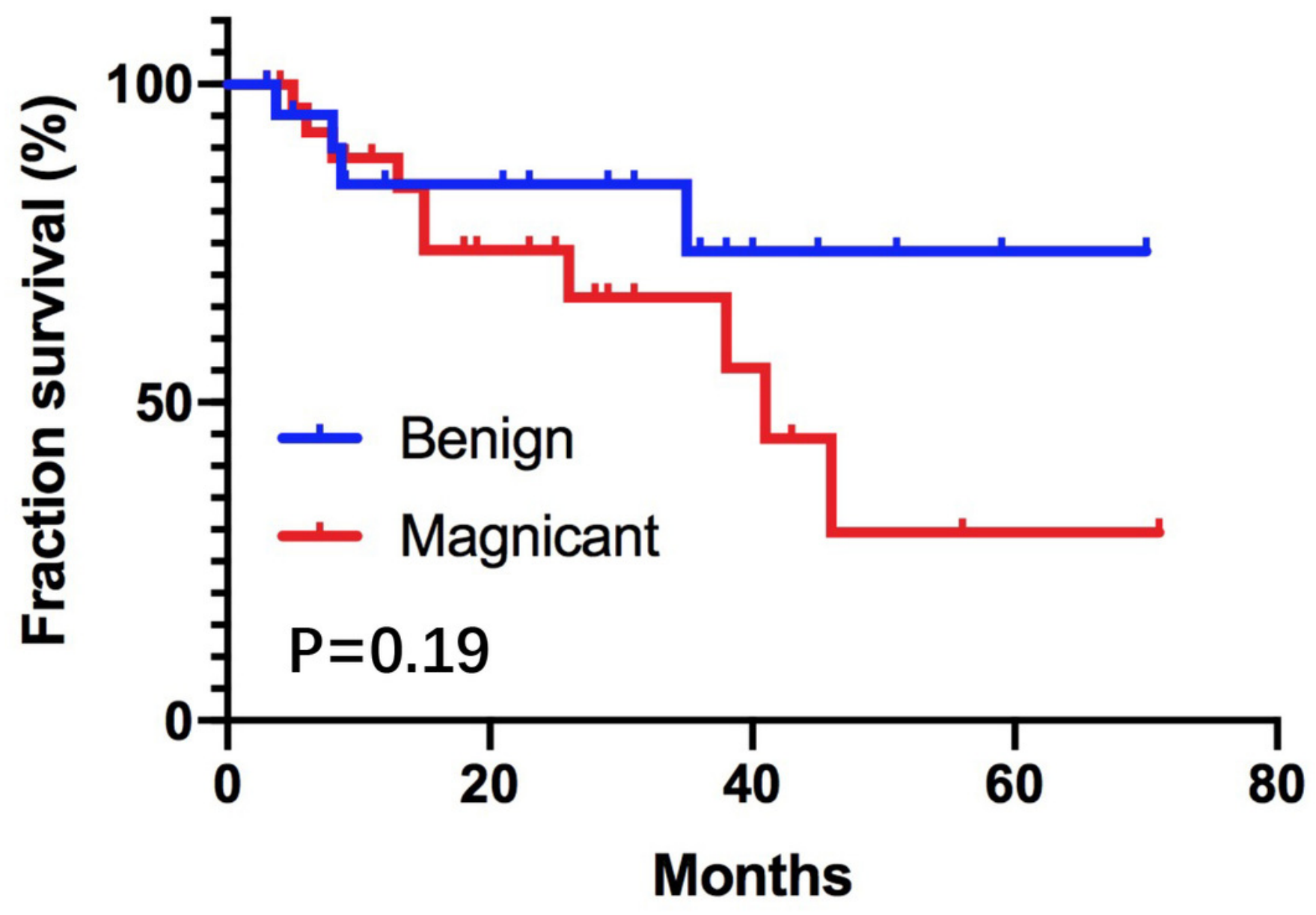




\section{Table $\mathbf{1}$ (on next page)}

Table 1 Clinicopathological parameters of 58 IPNM-B patients

Table 1 Clinicopathological parameters of 58 IPNM-B patients 
Table 1 Clinicopathological parameters of 58 IPNM-B patients

\begin{tabular}{|c|c|c|c|c|c|c|}
\hline \multirow{2}{*}{ Clinical Features } & \multicolumn{2}{|c|}{ Benign } & \multicolumn{2}{|c|}{ Malignant } & \multirow{2}{*}{$x^{2}$} & \multirow{2}{*}{$\mathrm{P}$ value } \\
\hline & $\mathrm{N}$ & $\%$ & $\mathrm{~N}$ & $\%$ & & \\
\hline Age (years) & & & & & 0.017 & 0.897 \\
\hline$\leq 60$ & 15 & 44.12 & 19 & 55.88 & & \\
\hline$>60$ & 11 & 45.83 & 13 & 54.17 & & \\
\hline Gender & & & & & 0.500 & 0.479 \\
\hline Female & 17 & 48.57 & 18 & 51.43 & & \\
\hline Male & 9 & 39.13 & 14 & 60.87 & & \\
\hline Stones & & & & & 0.012 & 0.913 \\
\hline Negative & 6 & 46.15 & 7 & 53.85 & & \\
\hline Positive & 20 & 44.44 & 25 & 55.56 & & \\
\hline $\begin{array}{l}\text { Previous biliary } \\
\text { surgery }\end{array}$ & & & & & 0.139 & 0.710 \\
\hline Negative & 11 & 47.83 & 12 & 52.17 & & \\
\hline Positive & 15 & 42.86 & 20 & 57.14 & & \\
\hline Jaundice & & & & & 0.330 & 0.566 \\
\hline Negative & 10 & 50.00 & 10 & 50.00 & & \\
\hline Positive & 16 & 42.11 & 22 & 57.89 & & \\
\hline CA19-9 & & & & & 0.051 & 0.821 \\
\hline Normal & 13 & 44.83 & 16 & 55.17 & & \\
\hline High & 13 & 41.94 & 18 & 58.06 & & \\
\hline CA242 & & & & & 7.083 & 0.008 \\
\hline Normal & 21 & 56.76 & 16 & 43.24 & & \\
\hline High & 5 & 21.74 & 18 & 78.26 & & \\
\hline CEA & & & & & 8.148 & 0.004 \\
\hline Normal & 25 & 54.35 & 21 & 46.65 & & \\
\hline High & 1 & 8.33 & 11 & 91.67 & & \\
\hline $\begin{array}{l}\text { Surgical method } \\
\text { radical resection }\end{array}$ & 22 & 47.83 & 24 & 52.17 & 0.808 & 0.369 \\
\hline
\end{tabular}




$\begin{array}{lllll}\text { palliative resection } & 4 & 33.33 & 8 & 66.67\end{array}$

$2 \chi^{2}$ test was used to compare the distribution of clinical features between benign IPNM-B patients

3 and malignant IPNM-B patients.

4 CA19-9 normal reference range: $0-35 \mathrm{U} / \mathrm{mL}$.

5 CA242 normal reference range: $0-20 \mathrm{U} / \mathrm{mL}$.

6 CEA normal reference range: $0-5 \mathrm{ng} / \mathrm{mL}$.

7 A P value $<0.05$ was considered significant. 


\section{Table 2 (on next page)}

Table 2 Multivariate analysis of factors contributing to overall survival in 29 malignant IPNM-B patients

Table 2 Multivariate analysis of factors contributing to overall survival in 29 malignant IPNM-B patients 

in 29 malignant IPNM-B patients

\begin{tabular}{|c|c|c|c|c|}
\hline \multirow[t]{2}{*}{ Variables } & \multicolumn{2}{|l|}{ Univariate analysis } & \multicolumn{2}{|l|}{ Multivariate analysis } \\
\hline & $\operatorname{HR}(95 \% \mathrm{CI})$ & $\begin{array}{l}\mathrm{P} \\
\text { value }\end{array}$ & $\operatorname{HR}(95 \% \mathrm{CI})$ & $\begin{array}{l}\mathrm{P} \\
\text { value }\end{array}$ \\
\hline Age $(<60$ vs. $\geq 60)$ & $0.114(0.002-6.305)$ & 0.413 & - & - \\
\hline Gender (female vs. male) & $0.166(0.012-2.216)$ & 0.838 & - & - \\
\hline $\begin{array}{l}\text { Stones } \\
\text { (negative vs. positive) }\end{array}$ & $2.007(0.166-12.283)$ & 0.540 & - & - \\
\hline $\begin{array}{l}\text { Jaundice } \\
\text { (negative vs. positive) }\end{array}$ & $3.890(0.138-25.550)$ & 0.074 & - & - \\
\hline $\begin{array}{l}\text { CA19-9 } \\
(\leq 35 \mathrm{U} / \mathrm{L} \text { vs. }>35 \mathrm{U} / \mathrm{L})\end{array}$ & $0.835(0.061-11.335)$ & 0.099 & - & - \\
\hline $\begin{array}{l}\text { CA242 } \\
(\leq 20 \mathrm{U} / \mathrm{L} \text { vs. }>20 \mathrm{U} / \mathrm{L})\end{array}$ & $0.326(0.063-1.678)$ & 0.116 & - & - \\
\hline \multirow[t]{2}{*}{$\begin{array}{l}\text { CEA } \\
(\leq 5 \mathrm{ng} / \mathrm{mL} \text { vs. }>5 \mathrm{ng} / \mathrm{mL})\end{array}$} & $1.143 （ 0.086-$ & 0.097 & - & - \\
\hline & $9.181)$ & & & \\
\hline $\begin{array}{l}\text { Lymph node metastasis } \\
\text { (negative vs. positive) }\end{array}$ & $0.368(0.054-2.519)$ & 0.015 & $0.442(0.111-1.755)$ & 0.246 \\
\hline $\begin{array}{l}\text { Surgical method } \\
\text { (radical resection vs. } \\
\text { palliative resection) }\end{array}$ & $5.444(0.337-27.848)$ & 0.018 & $0.081(0.010-0.673)$ & 0.665 \\
\hline $\begin{array}{l}\text { Differentiation degree } \\
\text { (high vs. medium-low) }\end{array}$ & $0.056(0.007-0.451)$ & 0.000 & $0.06(0.007-0.486)$ & 0.008 \\
\hline
\end{tabular}

3 Univariate and multivariate analysis of prognostic factors in 29 malignant IPNM-B patients

4 included in the survival analysis.

5 Statistical analyses were performed by Cox proportional hazards regression. A P value $<0.05$ 
6 was considered significant. Italic indicates significant $\mathrm{P}$ values.

$7 \mathrm{CI}$, confidence interval. 